\title{
Synthesis: Making magic with GenieMo
}

\author{
Luciana Haill \\ University of Brighton \\ Brighton, UK \\ lucianahaill@icloud.com
}

\author{
Nick Lambert \\ Ravensbourne University \\ London, UK \\ n.lambert@rave.ac.uk
}

\begin{abstract}
GenieMo is a real-time communication tool based on LiveScan3D, created to empower volumetric content creation and communication for social good. It presents a potentially democratising set of tools that can demystify technological processes that seem out of reach when presented by corporate "big tech". This paper explains how working with GenieMo develops some of the potentials in earlier works of telematic art, using a capture system, social hot-casting with additional real-time brainwave recording and visualisations to create something new that is also rooted in the history of immersive art.
\end{abstract}

\section{GenieMo. Telematic art. Volumetric capture. Brainwaves. Neurofeedback. IBVA.}

\section{INTRODUCTION}

In 2020 Playla.bZ founders James Edward Marks and Marius Matesan asked me to join their team as 'artist in residence' exploring and documenting their new volumetric capture, mixed reality system called GenieMo. I first met PlayLa.bZ in 2017 in their research space, the Holoportal, within Ravensbourne University London. This became an award winning, transformative creative space where students, incubation \& enterprise collaborate, for rapid prototype ideas with multi dimensional computer vision, emerging technology \& new creative media. They were interested in how I could combine this with my practice as a neurofeedback artist.

Following an initial meet up and the loan of a PC and peripheral hardware everything was actuated remotely due to the third Covid-19 restrictions. In 2020 for an event hosted by National Gallery X called Levitations I explored brainwave feedback negotiating nonlinear narratives in a new artwork called 33: $E E G$ encompassing metacognition in the form of reflective thought with some attempted control to livestream 'final-cut' of dreamlike videos I had assembled. This was an extension to flowstate techniques I learned in 2007 in special Lucid Dreaming workshops in Hilo, Hawaii led by $\mathrm{Dr}$ Stephen LaBerge. We practiced techniques to increase our critical reflexive attitude, teaching us how to attain lucid dreams involving developing insight, control, and witnessing - a softened awareness process. These are three defining criteria of lucid dreams. I continue to also employ neurofeedback training for Alpha-Theta brainwave activity to encourage intuitive thinking, rewarding the increases with instantaneous positive audio feedback, a methodology I use when approaching new works. Neurofeedback training has been proven to enhance attention, memory, learning, micro-surgical skills, mental rotation, intelligence, sleep, and well-being in healthy participants.

The implementation of medical interfaces for both personal transformation and artistic expression is something I have over twenty-five years of experience with. My research and practice centres around the acquisition of electrophysiological data (the EEG signal), investigating different interfaces and the implementation of 'EEG' signals in both creative and scientific context. Artistic and scientific experimentation with EEG has been happening since the late 1960s as artists Alvin Lucier, Pierre Henry, Richard Teitelbaum and David Rosenboom were experimenting with EEG feedback. A collaboration between London Fieldworks (Bruce Gilchrist and Jo Joelson) and internationally celebrated artist Gustav Metzger created Null Object (2012) in which a robotic arm carves out of Portland stone a form controlled by Metzger's brainwaves as he attempts to think about 'nothing'. I have employed portable brainwave monitors, particularly the Interactive Brain Wave Visual Analyzer (IBVA) a frontal lobe EEG monitor, since the 1990s when I studied the first degree in Interactive Art in Newport, University of Wales, with Roy Ascott. I was enthralled by Ascott's enthusiasm about cyberneticians and his vision of a networked community with the potential global authorship (prior to general public access to the World Wide Web). Roy Ascott expresses in The 
Telematic Embrace -'Instead of the artwork as a window onto a composed, resolved, and ordered reality, we have at the interface a doorway to undecidability, a dataspace of semantic and material potentiality'.

The IBVA software allows one to see and monitor visual representations of the beta, alpha, theta, delta, and gamma frequencies of brain waves, as well as the coherence between the left and right frontal hemisphere signals. The IBVA system has been used in publicly engaging, interactive brainwave triggered art works notably by Mariko Mori in 'Wave-UFO' a large-architectural sculpture that has extensively toured. I attended this experience as a friend of the inventor of the IBVA, Masahiro Kahata when he was working on this in the Venice Biennale 2005. Using the IBVA system in the development of brain games for e-learning has been published by artist Paras Kaul and presented at conference venues, such as the Special Interest Group for Computer Graphics and Interactive Techniques (SIGGRAPH) in the United States and the Dana Centre Museum of Science in the UK. It has also been used for research conducted by Dunne and Jahn in the Engineering Anomalies Research Laboratory.
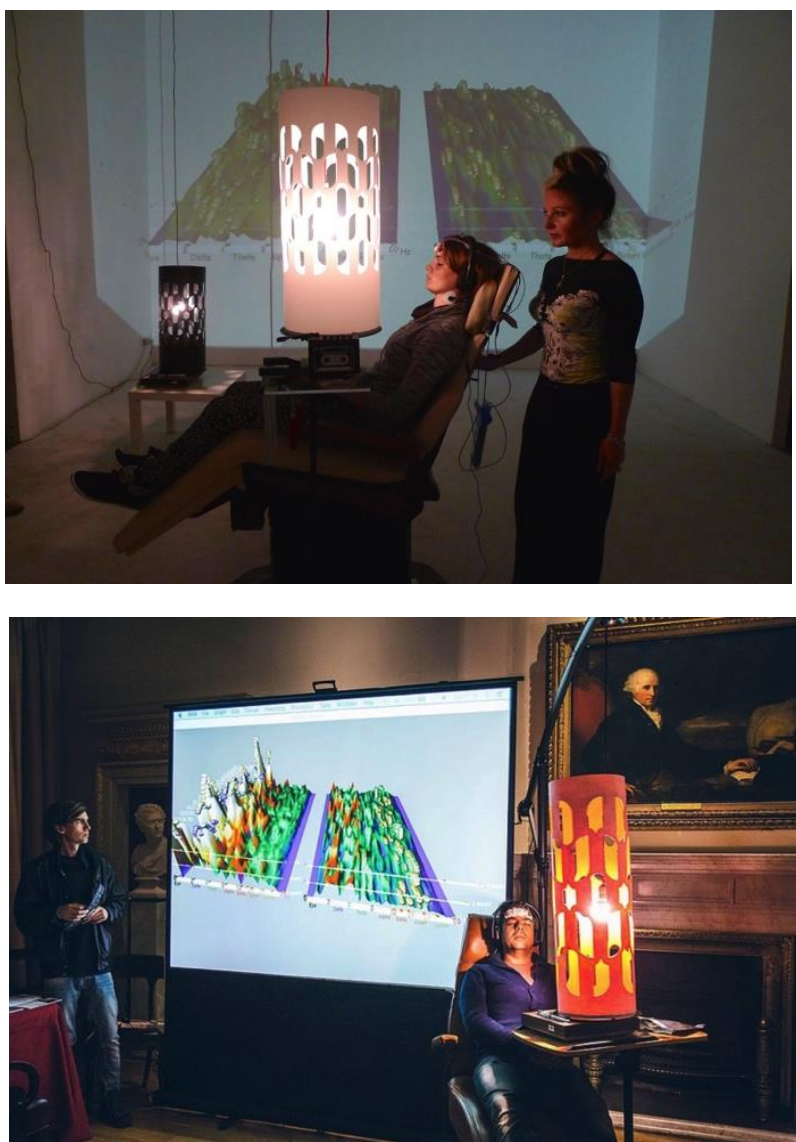

Figure 1. Luciana Haill and the DreamMachine showing EEG output.

\section{ABOUT GENIEMO}

In 2020 all work and projects transitioned into an array of contingency plans:hybridised versions and postponements due to the rules and regulations imposed by the government during a global pandemic we refer to as Covid-19. It called us to question what being 'live' really means, more than an online presence and how artists would ever interact with an audience again - from the daily necessities to social and cultural events. Galleries and institutions attempted to adapt with new social distancing rules in place, but theatres, clubs, temporary live music venues \& the performative arts experienced more restriction as no direct human contact was permitted. Normally this would not be an inspiring starting place for artists exploring a fusion of 4D camera vision \& engagement through mixed reality experiences and their potential to reach beyond the arts.

PlayLa.bZ Chairman and IBC Future Zone \& Society of Motion Picture \& Television Engineers (SMPTE) fellow Professor David Crawford explained: "This '3D capture' facility is really the next step in technology for communications \& social media..."

PlayLa.bZ has been working on this technology for several years. James Edward Marks and Marius Matesan were inspired by an iconic scene in the first Star Wars film when Princess Leia pleads for help, projected as a small 3D hologram - an 'augmented reality volumetric selfie' prototype. So they developed a solution that could democratise the implementation of this concept without too much potentially costly and complex equipment.

In July 2017 their early research demonstrated how quickly the technology was moving in terms of accuracy in placement and tracking, this was the effective break away solution that became an early concept version GenieMo. A video recorded in November 2019 showed GenieMo developer

Matesan guiding a researcher in the use of the technology. Even on a 2D screen watching the demonstration on YouTube years later, you still get that initial sense of wonder as you see the researcher's head moving in 3D space and move around and behind the actual person in apparent real-time.

In 2020 the team made 'GenieMo' as hardware agnostic as possible, so it does not involve any proprietary software and above all, it is an easy-touse solution. It is not reliant on $5 \mathrm{G}$ technologies, and it only needs one sensor to be effective and reliable for various applications. The software is free, in line with Harry Halpin's dictum: "Free software is a political programme that goes beyond 
just 'open source' and 'open access' to code, although it provides open access to code as it is necessary for freedom."

The latest version of GenieMo is compatible with Sketchfab and Abstract LiveScan3D, a free, open source system for live, 3D data acquisition that no longer requires multiple Kinect v2 sensors connecting to the latest model or equivalent spatial Al camera module system (such as the OAK_D using the OAK API system). GenieMo supports imports from 40 other 3D model formats; it can capture real-time video, record sound from multiple microphones; capture 360 video and multidimensional mesh all at once. It has a 'one click' real time 4D social-cast video communication and mixed digital media performance utility, and can stream 360 video \& cross platform play in real-time through social-casting.

Using the LiDAR sensor that emits a single short laser pulse also removes the need for a physical green-screen via the spatial auto cutout of background. One of the greatest features of the GenieMo (MdMA) System is that it is completely mobile and usable in any environment. Because of the system's versatility it can be deployed in any environment safely and economically, it is not much larger than a hand luggage suitcase including a computer such as an INTEL fanless NUC mini PC model powerful

The new Kinect camera is commonly employed by applications in volumetric filmmaking and journalism, interactive installations, advertising communication and by some artists. It is no longer designed to interface in the world of gaming in the way the original Kinect camera did with X-box, it has been clearly been repurposed by artists. Performance and body projection mapping artists Kimatica always use $X$-box Kinect in their transcendental interactive artworks. 'Shadows of Light' made by artist Alex May was first shown in The Kinetica art show in London in 2011. It is a contemplative interactive digital video installation using the original Kinect camera, exploring the concept of "slow interaction" where the visitor is rewarded for standing still.

Simultaneously as part of PlayLa.bZ's InnovateUK grant, GenieMo is being interrogated by world leading 'virtual surgeon' Professor Shafi Ahmed involving telepresence in addition he works wearing HoloLens 2 - a pair of mixed reality smartglasses. Using GenieMo with the Hololens display (recording and viewing close proximity in the real world) and transmitting the first person view, is an excellent training tool for the medical students increasing doctor to patient communications. outside of operating theatres and hospitals. He has also added the 'LookingGlass' volumetric display that offers a good depth of field visual display for surgical training sessions to his medical students remotely.

GenieMo permits a seamless media-mixing playground combining a computer's Virtual Camera with inputs from SPOUT, WebRTC \& WebSocket network protocols. I wanted to incorporate live brainwave signals as they are being analysed by IBVA software on my Apple Macbook Pro, networked to the NUC PC running GenieMo.The spatial auto cutout of background enabled me to appear as if I was in a room - the 'context' decorated by my realtime interactive brain waves projected onto every surface of a cube. The SPOUT permits connection of an external (using a free tool from NewTek NDI (Network Device Interface) video stream sent via the Open Broadcasting System OBS software, also free.

During subsequent meeting with Playla.bZ using GenieMo social casting function I was able to appear on the screen to all the other people present in the online meeting space as if I was sitting in a studio within my own brainwave biofeedback, in the context of a reactive virtual environment where the wallpaper was providing a level of visual neurofeedback! I initially conceived of a series of networked installations (between phases of localised lockdowns ) occurring in London and Brighton and beyond - wherever we could add a social hot casting post with a suitable camera. But the restrictions continued and this meant in fact I had to work alone with updates handled via online meetings. This also meant virtualising the interactivity (which should have involved connecting the audience through one to one engagements in real life locations) with a brainwave EEG monitor.

Inside GenieMo social hot-casting to engagements in other locations I developed a sense of a double presence, the screen now acting like a cameramirror, in my home surrounded by decorative mirrors. My voxelated face requires uniform white lighting to guide the edge detection $\mathrm{Al}$ and avoid over stretching \& cyber-dysmorphobia. I began to apply makeup more dramatically when presenting in a group Playla.bZ meeting to accommodate the brighter lights, somewhat like a 1920s black and white film actor would have done. I remembered that artist Allan Kaprow stated in the 1960s:'The world would make up its own social relations as it went along! EVERYBODY IN AND OUT OF TOUCH ALL AT ONCE!' describes very well what a daily appearance in voxels feels like, challenging and empowering the technology

Gradually hauntological melancholia tainted our engagements. The term 'hauntology' was originally coined by Jacques Derrida in Specters of Marx, 
reappeared much in 2006 and was readopted by philosopher and writer on popular modernist culture Mark Fisher from his Warwick and Goldsmiths locations. 'Skyping' ceased (even sounding dated) - you wouldn't have attended a private view and party enabled on Skype for instance. It seemed to be engulfed by a myriad of other virtual meeting places, and even using Zoom as a verb invariably calls to mind a tiring screen-mediated method, life constrained by social distancing requirements. I was aware I should be embracing the hitches and glitches the way artist Nam June Paik described 'The intersection of experimental art with still nearly experimental technology produces all the hitches, glitches, delays, and improvisations you'd expect.' when he had to deal with the events in the "global disco" he'd built out of the latest electronic media technology.

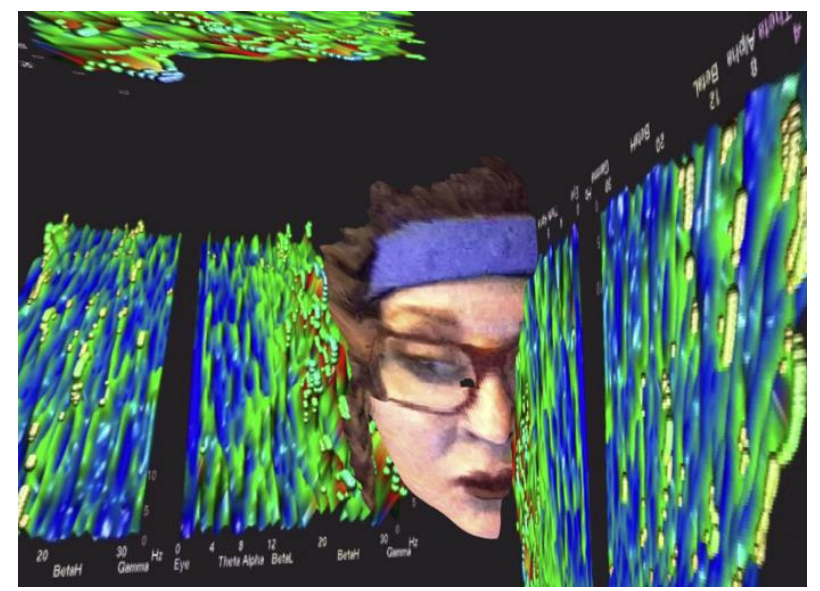

Figure 2. The artist captured as a voxel image inside the GenieMo-based artwork.

\section{INTO THE ROSE GARDEN}

"Time present and time past

Are both perhaps present in time future,

And time future contained in time past.

If all time is eternally present

All time is unredeemable.

What might have been is an abstraction

Remaining a perpetual possibility

Only in a world of speculation.

What might have been and what has been

Point to one end, which is always present.

Footfalls echo in the memory

Down the passage which we did not take

Towards the door we never opened

Into the rose-garden. My words echo

Thus, in your mind.

But to what purpose

Disturbing the dust on a bowl of rose-leaves

I do not know.

Other echoes

Inhabit the garden. Shall we follow?"

- T. S. Eliot Four Quartets.
I have always been perturbed with the concept of limbo, having been raised as a catholic - it is a place dead people spend time, after their lives have ceased. This has kept me awake as a child with fear, however when I contemplate spending time in telepresence, endlessly I feel less anxious. When I first read this poem by $\mathrm{T} S \mathrm{~S}$ Eliot it soothed my agnostic concerns. Particularly:

\section{'Other echoes}

Inhabit the garden. Shall we follow?"

Spectral, telematic echoes, voxelated dust particles, echoes \& glitches - inspired my initial version of a volumetric garden, where I am an echo. 'Into the rose garden' is an effort to visualise human thoughts where technology and art combine to render the unseen in an immersive spatiotemporal performative telematic artwork that exists in a livestream (broadband is essential for an acceptable frame rate of the EEG).

The work fuses mixed media, mixed reality \& telematic art research, practice and theory. Time passing is spent reading, playing piano \& upright bass in a 4D telepresence, the artist appears within a stage of their animated live EEG signals. Here is symbolic potential for transcendence - 'into the rose garden' - how do we 'get into' it? GenieMo's volumetric capture and spatial cut off provides a hidden space, a trap door in the stage. In the last year of extended lockdowns in our homes we have changed how we feel about time. Is this frameless place somehow timeless? Constrained by linear concepts of time keep the present moment as the focus - we obsess about the conception of the present.

Maybe Eliot is provoking us and Roy Ascott develops this further when he describes 'a doorway to undecidability, a dataspace of semantic and material potentiality' in the Telematic Embrace, He provokes us to think about an interpolation of all time, and every possible narrative, evolving a symbol of infinitude behind closed eyes. It captures a hauntological quality that can be grasped but never fully realised, the rose garden is not a painting of any still life, it is a suggestion of what our lives could be, in times where covid lock-down constraints have made the sweet downtime of freedom into a regimented, accountable chore. 


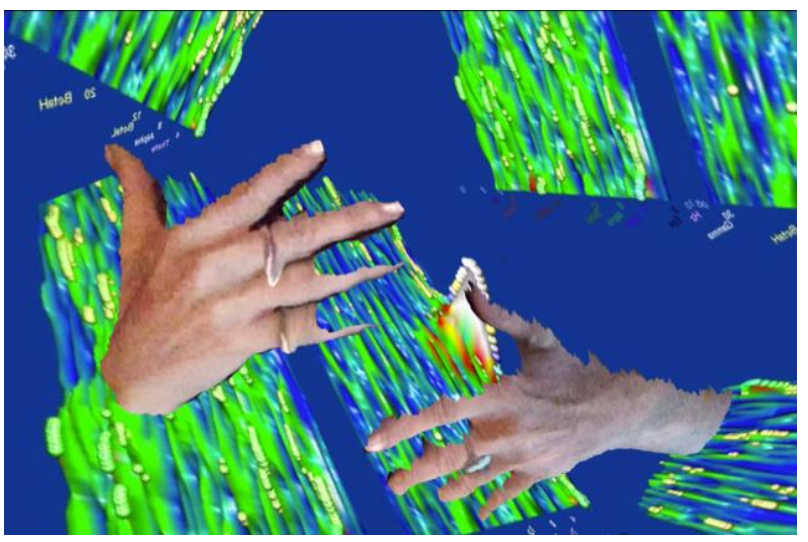

Figure 3. Into the rose garden sequence.

Taking inspiration from pioneers of interactive art, I imagined future fictions of how Alvin Lucier, Paik \& Charlotte Moorman (who place themselves at the centre of their interaction) would engage in GenieMo telepresence. 'Music for Solo Performer' by Lucier in 1965 involves EEG monitoring from the back of the head, the occipital region and the is exclusively based on Alpha brain waves, alpha rhythms or alpha synchronisation. Controlled mainly by closing the eyes, and is not so suitable for GenieMo as a technique, but could lead to a recreation of the compost's seminal experimental piece.

Next I took into consideration how this would be different to the remote engagement, in 'Telematic Dreaming' by Paul Sermon, where the telematic interaction was made complete by projecting a person from one location onto a bed in the other location - the gallery, permitting the audience to position themselves more or less inside this networked scenario. Two publicly accessible locales were interconnected through the experience. Some people cuddled up to their distant connection, aligning body parts to further touch or nestle into a virtual human in realtime. Originally I hoped to make a multiplicity of reciprocal transformations but these elements of engagement that are currently missing.

The connection facilitated by GenieMo's 'social casting' function into Google Meets where other participants 'the audience' appear reductive in 2D tiled gallery view on a screen, and currently due to covid-19 lockdown, they are most likely at home. Mediated through a monitor is not the desired view, the next stage as lockdown restrictions ease in the UK and social distancing rules are relaxed is to develop the piece through IRL \& url interactions. This limitation raises the question around if 'home' becomes a performative / gallery space. It appears for now that the telematic engagement is even more disconnected than being in a gallery. I realise what I want to achieve when I test the work is hybrid virtual physical experience where the public are allowed to explore the piece and see themselves becoming part of the screen as well as (or instead of ) watching it through a social media presence live-streamed.

Into the rose garden - Luciana Haill

The Playla.bZ team and I experimented with emulating the appearance of passing a cardboard VR viewer between our three screens in our separate spaces / GenieMo contexts in a video meeting, treating the camera just like a mirror. A link to this in included in here INSERT (youtube link $\&$ still screen grab)

The social cast function enables a different type of audience, where I can export my 4D volumetric self into Google Meetings or Youtube currency, although only those with the right hardware can be 'present'. And even when we do meet together we appear distanced. If another person is present you can import their window source into your background by using the "spout" tool. They can be in a 2D plane like a poster, a 360 'sky' which wraps around and requires size readjustment in an intermediary live video manipulation software such as VDMX, a sphere bubble - which has a good aesthetic for performance or the 6 plane cube that I prefer using.

When I first experienced myself inside a telematic space with the breakthrough moment of achieving the EEG over the LAN via OBS into GenieMo I was excited and disturbed together. My face voxels stretched as the light did not reach the outer contours enough giving me a strong sense of body dysmorphobia, not something $\mathrm{I}$ am ready to embrace in the work. The edges of my face mesh especially around the eyes were slanting into the invisible background, creating a new elfin cyberself. My fingers could point further along the upright bass strings than possible defying biology and appeared witch-like. These distortions and their associated unsettling could of course be exploited in an artform as well as the ability to select a rotate function that slowly allows once face to rotate 360 so you view it from inside, a dark side of the moon reversed illusion. The camera can appear to 'see' behind you when its stationary due to the 1-MP depth sensor with wide and narrow field-of-view (FOV) options and its 12-MP RGB video camera aligned to the depth stream.

To achieve this local area network between a PC running GenieMo with the Kinect and an EEG monitor transmitting brainwave information by bluetooth to the system which consists of a Mac running proprietary Interactive Brain Wave Visual Analyzer (IBVA), an EEG biofeedback system. The analysis software sends data to bespoke audio unit plugins communicating in realtime with third party audio applications Ableton Live (or Garageband - 
included free with OSX) and commercial video mixing software (VDMX). Open source broadcasting software OBS with NewTek NDI compiles this and sends via local network ethernet connection to the PC running GenieMo, receiving the brainwave live animations via an 'NDI spout' free plugin. The spout form becomes the stage and the one selected is an open box - chosen to subtly pay homage to Enrico Prampololinis avant-garde scenotechnique exhibition of 1936 , as a classic polydimensional Futurists' stage. Inside GenieMo there are other different contexts can be selected for scenes such as a forest or other 3D imported Unity 3D assets in a stage such as Bladerunner to appear within, or 3D worlds shaped into wraparound sky can receive the transported video sent from the Mac.

I further exploit the volumetric capture and the spatial auto cutout of background to enable objects to be passed to me from behind me that the camera cannot detect. The first object is a red book, I was thinking of Jung's red book, although I do not possess this magnificent item, as a stimulant for a brainwave reading. To be positioned in GenieMo geometry initially on a x-axis of the brainwaves, as if sat on a magic carpet of my own EEG calm with few peaks, and as the book is handed to me (by an assistant) invisibly from beyond the camera's depth of field, I begin reading his personal journey into his own consciousness and psyche, will this elicit more intuitive Theta Brainwaves? Another red book is famously the one handed to the guest on each show that used to run on broadcast ITV 'This is your life' and would contain memories and nostalgic anecdotes from their lesser known past.

The theme tune to this programme is one I have played over 500 times on the piano \& bass in the last year. I have a fondness for library music and especially those that have hauntological connections, expressing variations whilst interpreting the original composition called 'Gala Performance' by Laurie Johnson' it has become a compulsive variation on a theme. Whilst I am sat at at an upright stick bass or the piano, another scene in this live artwork will be my playing this tune in modes both of "becoming" and expressing steganographic "informatics of domination" which could evolve into a multiverse of brainwaves. The original scene is myself reading silently the last part of the Four Quartets in 'Burnt Norton' by TS Eliot where the title of my work is inspired from. An assistant is needed to reposition the volumetric video captured within the context spout, using a new 'grab' function that makes it easier to rotate the context around the fixed video image, using repositioning views from above, left and right.

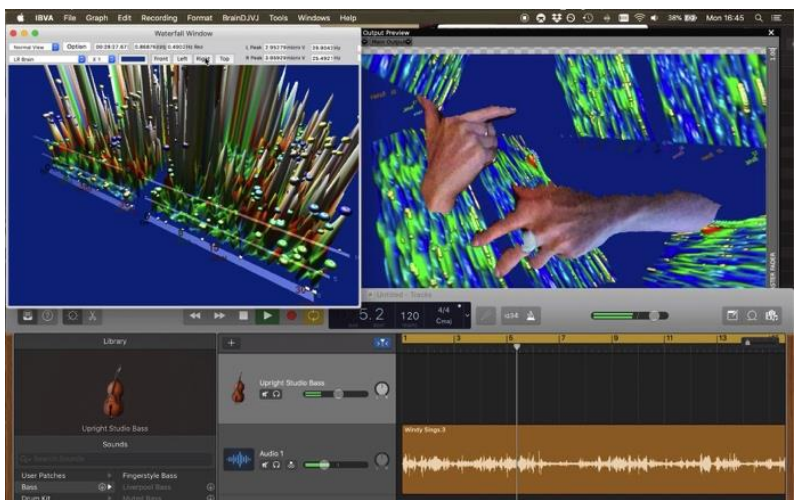

Figure 4. Into the rose garden, sofware IBVA, Garageband and GenieMo live volumetric video capture.

Whilst playing or reading the artist is connected to an EEG monitor that can express changes in the note when running Ableton Live at the same time. Using the IBVA the EEG data from 0 hertz to around 60 hertz is relayed to an audience via speakers and data projectors, which are replaced now by GenieMo, computer screen and headphones.. The amplitude in microvolts and the speed of all the brainwaves in Hertz are detected using 5 electrodes in a headband across the forehead, above the eyebrow. These are at Fp1 \& Fp2 on the 10-20 System of Electrode Placement. The signals are sent by IBVA software to custom audio unit plugins installed in the library of Mac for use by other sound software. They enable multiple different EEG modulators to effect thresholds in realtime a multi layered soundscapes. relatively simple algorithms and spectral features, including FFT, EEG band powers. I aim to create a heightened 4D awareness of my body as a site of consciousness and of the experience and sensation of consciousness occupying space.

In GenieMo I can create an evocative demonstration of this perception of consciousness. EEG is a non-invasive imaging technique which is widely used in scientific studies and which displays activity generated by structures inside the brain by recording activity from the scalp's surface via electrodes. Combined with neurofeedback (the ability to voluntarily use learned control of one's own EEG activity) in the brain as proven by Joe Kamiya in the late 1960s. I have been implementing EEG signals within my artworks since the late $90 \mathrm{~s}$, and I use a system I have been involved with the software development side known as the IBVA. It is a compact sub- $\$ 1000$ noninvasive consumer EEG headset with bespoke software system, it includes 2 channels using 5 electrodes for prefrontal lobe monitoring, positioned on the forehead above the eyes. Based on the 1020 International electrode placement convention, the pre-gelled disposable electrodes were located at FP1 FP2 using an ear-clip as the reference 
electrode. It is in the same bracket of low cost EEG devices including the InteraXon Muse, the Neurosky MindWave, the Emotiv Epoc, the Emotiv Insight, and the Open $\mathrm{BCl}$ widely-used commercial models.

IBVA unique hardware and software interface for Apple OSX shows the brain's left and right prefrontal brainwaves as signal frequency content divided into the following clinically relevant frequency bands: alpha $(8-13 \mathrm{~Hz})$, beta (14-30 $\mathrm{Hz})$, and theta $(4-7 \mathrm{~Hz})$ waves. When comparing the power spectra, the alpha and beta waves decreased when drowsy, while the theta waves remained constant.

"Time moves in one direction, memory another. We are that strange species that constructs artifacts intended to counter the natural flow of forgetting."

\section{- William Gibson, Distrust That Particular Flavor}

\section{CONCLUSION}

"The difference between inner experience and philosophy resides principally in this: that in experience, ... what counts is no longer the statement of wind, but the wind."

Artists have championed the need for connection with other humans, particularly in this age of digital technology affected by the covid-19 rules regarding social interactions. Computer-mediated telecommunications networked art and futuristic visions such as Allan Kapprow's idea of TV arcades in the 1960s predicted 'People will be free to do whatever they want and will see themselves on the monitors in different ways. A crowd of people may multiply their images into a throng.' They predicted the human need for an emergent social media enabled through telepresence.

In these pioneering works from the late 1960s through to the $90 \mathrm{~s}$, it is not the desire that is outmoded, but in the passing of time the cutting edge electronics that ultimately enabled it also superannuate the art. Nam June Paik's "global village" echoed Marshall McLuhan's pronouncement that the artist prophecies the "message of cultural \& technological challenges decades before the transformational effect occurs" - he wanted artists to help develop a more democratic mass media, predicting the world wide web in his internet superhighway vision. He also wanted to harness technology for aesthetic \& political change. In 1969 Allan Kaprow delivered 'Hello' within an interactive TV happening called 'The Medium is the Medium'. Kaprow was interested in the idea of 'communications media' as non-communications, and 'Hello' dismantled the disruptive manner by which technology mediated interaction.

Telematic performances open up artists' work to whole new audiences - connecting them both in person and online, using desk mounted, projected, head-mounted or discretely augmented and mobile forms of connection. GenieMo is part of this reemerging wave of artistic experiences, networked adapted into telepresence, and media, agnostic. It facilitates the stage, the club or the activist rally that connects people in a networked disruption to normality. As a rapid prototype it presents a potentially democratising set of tools that can demystify technological processes that seem out of reach when presented by big corporations or other entities with business plans around it. The software is of course free and all the hardware needed to run it costs under $£ 1000$ including the PC (such as the compact, portable Intel NUC model already mentioned.) A future addition (dependent on the next injection of funding) will be GenieMo connecting with $\mathrm{Al}$ and standard streaming RGB mobile cameras with $\mathrm{Al}$ that are coming soon.

In March 2021 I performed in 4D using GenieMo social-casting to Youtube 'Live' in the virtual music room of MozFest delivered into the VR chatroom platform called SpatialChat. It permits up to 50 guests to be in the same party / meeting space, and a relationship to each guest's location and proximity is expressed relative by their volume, enabling guests to congregate closeup for discrete conversations. After two rehearsals ahead of the 'Cultural Party' the experience felt much like a IRL event. As I was the only person with a volumetric camera as I performed this reinforced the need the democratisation of the volumetric capture camera technology that enables the audience and other art centres, community hubs or collaborators to join in. A similar example of a device commercially available adding volumetric depth is the 'SubPac' phenomenon, feeling sound achieved by the wearable speaker delivering adjustable levels of sub-bass vibrations for DJs, domestic, cinematic and expansive sound Art experiences. The cost of a SubPac and a Kinect camera are almost identical.

Explorations into the artistic and philosophical possibilities happening in emerging digital technologies are accelerating achievements so that we may experience new meaningful transcendent interchanges within a telematic environment. GenieMo is both a system in my home and also an enabler for my artistic telepresence, it is a one click to live-stream via broadband, when I chose to social cast myself as voxels \& brainwaves. I foresee it will be the chosen, not the contingency option in creative immersive delivery in the future. 
To refer once again to TS Eliot's Burnt Norton prior to the mention of a rose garden :

'What might have been is an abstraction

Remaining a perpetual possibility'

I feel it is here that my audience connects to me, and I connect to them, I however need another stage to complete the telematic artwork in a public space. Each day I spend time here, it raises the question - am I only performing when I connect from home to a telematic place, then I am truly living in the art. In the same way as avant-garde TV Bra cellist Charlotte Moorman wholeheartedly described - how she is intrinsic with the tools \& technologies, which enable her art "The bra is one third of it, I'm one third of it, and my cello is one third of it. When we're all together, the work is complete." This is maybe a museum or curatorial legacy issue, who wonders if computers and Al cameras will become relegated as relics of past performances, consigned to storage.

It has been over 25 years since artist Char Davies' ground-breaking VR experience Osmose was live. Requiring the 'immersant' to use their breath as a navigation tool, wearing a bulky 'HMD' as now there are much lighter and much less costly systems available commercially. She explained "..the future of the technology is now controlled by a few immense corporations whose priority is making money. Increasingly, the means of production and the means of delivery are being centralized and monetized.

As Covid-19 continues to impact our reduced social and cultural experiences, we also witness the rapid decline of IMAX 3D cinemas we see the rise of affordable consumer VR devices. Artists have a role to champion public concerns that have been proven real by the totalitarian control Facebook has with the subsidised (and seductive) VR Oculus Quest2 device that allows users and consumers to immerse and create. In 2021 Google made its virtual-reality painting tool open source, discontinuing work on the app - but making the code publicly available. The Tilt Brush app has a community of artists that specialise in this medium that allows users wearing VR headsets to "paint" sculptures in three-dimensional space. This is a major selling point for artists as an Oculus Quest2 costs approximately £300, and now programmers can bring in new features, which could lead to several alternative and competing versions.

Too often technological creativity can be turned into a commodity primarily oriented toward research and intellectual appropriation by new corporate regimes. To avoid this some artists are making work as resistance, an example of this is a video installation by collective Hyphen-Labs The Gospel According to Yawn (2019) commissioned by Somerset house, is demonstrating how the act of yawning to stay awake is the last bastion of resistance to our 24/7 world. The photo-booth installation encourages yawning, inside participants are confronted with pictures of people yawning (we easily mimic this precursor to sleep behaviour), if they can't resist they will also be captured midyawn, and the art achieves an act of resistance. Luis Suarez-Villa describes how human creativity and technology are exploited by the new corporatism, arguing that new forms of democratic participation and resistance are needed, if the social pathologies created by this new version of capitalism are to be checked. These views are shared by PlayLa.bZ founders.

Born out of necessity in keeping performative arts alive, new telematic hybrid artworks could potentially pave the way for future mixed reality performances to co-exist alongside live, in-person IRL event. Right now we still can't share interface hardware (even headphones) in installations, artists can make works adapted into hybrid virtualphysical events using telematic \& live-streamed platforms, which widen the audience accessibility. This can raise important questions about what it means to 'be live' whilst blurring boundaries 'in realtime.' A relationship is evolving through social media and video meeting platforms about how to deliver live art, exploring intersections, blindspots, anomalies whilst observing intimate interactions. When the audience is watching in private, can their perspective be made more meaningful?

Futurologists predict that 3D videos will be the dominant traffic in the fastest data networks in the Metaverse of the '20s. Embracing experimental technology to bring to life a show in March 2021 is The Royal Shakespeare Company (RSC) recently premiered Dream enabling a smartphone ticket holder to interact with performers involving the UnREAL games engine in the production. The RSC isn't the first theatre company to create a digital experience combining gaming technology with live theatre. Les Enfants Terribles, Swamp Motel and Darkfield are among those to have successfully delivered lockdown productions that fuse these entertainment genres. Dream is one of four Audience of the Future Demonstrator projects, supported by the government's Industrial Strategy Challenge Fund. The production has been created in collaboration with Manchester International Festival (MIF), Marshmallow Laser Feast (MLF) and Philharmonia Orchestra. 


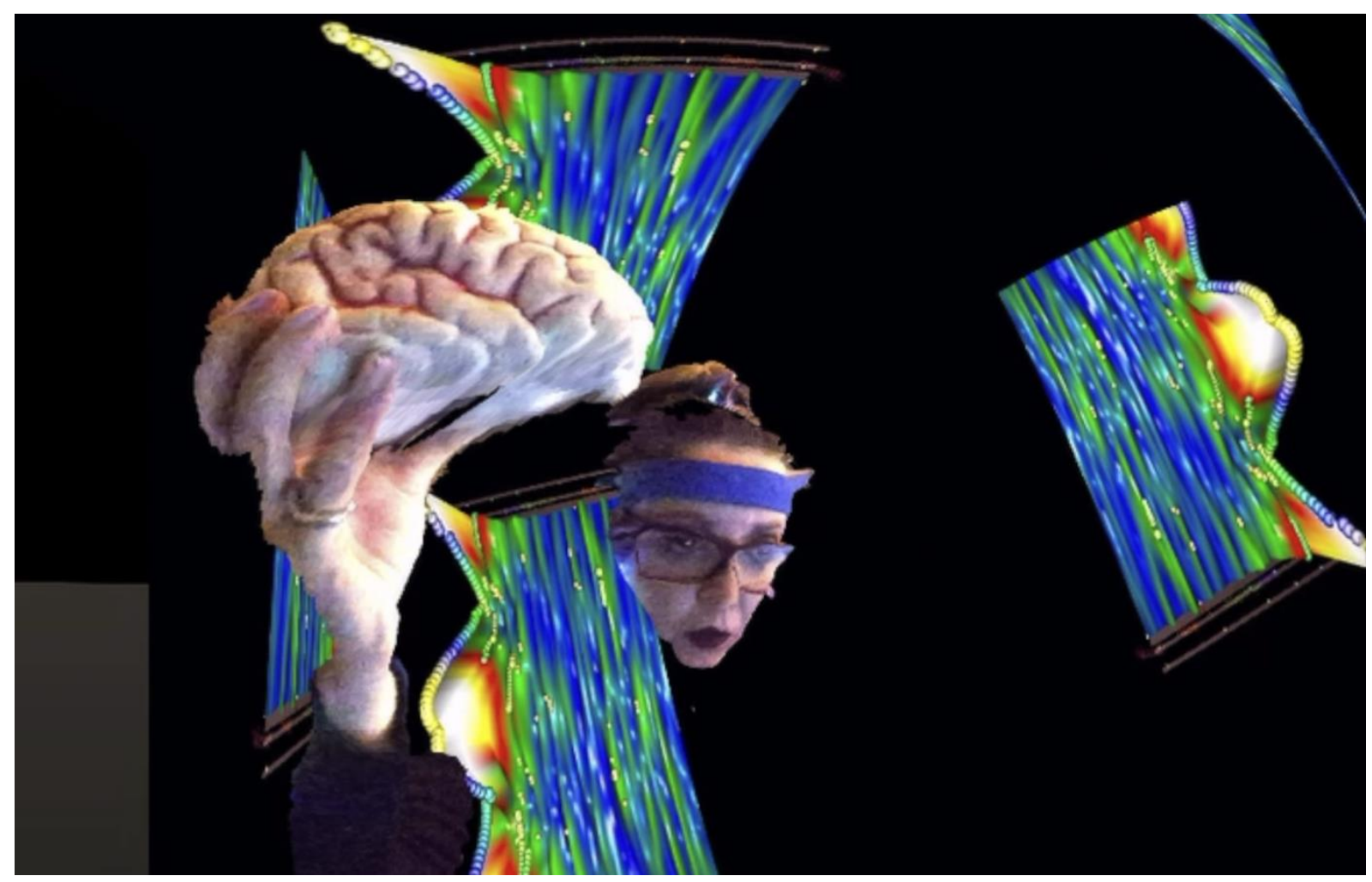

Figure 5. Into the rose garden sequence.

Self-service SAAS [software as a service] platforms could also enable art to be more accessible to a wider audience, available to anyone, anywhere in the world. In 2020 the idea of sharing screen time with others outside of overseas Skype business calls for business irrelevant and avoidable. This also affects art that involves realtime biofeedback signals because no one else can be in the cockpit position wired up to the interface for now. GenieMo has the potential to alter the way we experience the world around us, it can add multidimensionality into interactive artworks infusing multiplicities into engagements, confusing user recognition (in a way that is not yet fully realised) in telematic mixed realities via social casting may potentially be a useful addition in certain talking therapies that are achievable remotely. Unfiltered representations of the self in multi-dimensional casting, with splintering edge detection, without filter masking may look brutal, yet for me this generated a nostalgic yearning for a presence and immediacy that never has existed and may never will.

'Point to one end, which is always present.
Footfalls echo in the memory
Down the passage which we did not take
Towards the door we never opened'.

\section{REFERENCES}

Ascott, R. 1990. "Is There Love in the Telematic Embrace?" Art Journal. New York: College Arts Association of America. 49:3

Lucier, A. 2001. Seminar. In D. Eibenová, M. Perry and J. Novak (eds.) Ostrava Days 2001: New Music Institute and Festival. Ostrava: Ostrava Center for New Music, 27-38.

Rothfuss, J. 2014. Topless Cellist The Improbable Life of Charlotte Moorman. MIT Press. 